OPEN ACCESS

Edited by:

Tammy A. Morrish,

Independent Investigator,

United States

Reviewed by:

Feng Zhang,

Fudan University, China

Dustin C. Hancks,

University of Utah, United States

Yong Zhang,

Institute of Zoology (CAS), China

*Correspondence:

Ryuichi Ono

onoryu@nihs.go.jp

Specialty section:

This article was submitted to

Cellular Biochemistry,

a section of the journal

Frontiers in Chemistry

Received: 22 December 2016

Accepted: 23 May 2017

Published: 15 June 2017

Citation:

Yasuhiko Y, Hirabayashi Y and Ono $R$

(2017) LTRs of Endogenous

Retroviruses as a Source of Tbx6 Binding Sites. Front. Chem. 5:34.

doi: 10.3389/fchem.2017.00034

\section{LTRs of Endogenous Retroviruses as a Source of Tbx6 Binding Sites}

\author{
Yukuto Yasuhiko, Yoko Hirabayashi and Ryuichi Ono* \\ Division of Cellular and Molecular Toxicology, Biological Safety Research Centre, National Institute of Health Sciences, Tokyo, \\ Japan
}

Retrotransposons are abundant in mammalian genomes and can modulate the gene expression of surrounding genes by disrupting endogenous binding sites for transcription factors (TFs) or providing novel TFs binding sites within retrotransposon sequences. Here, we show that a (C/T)CACACCT sequence motif in ORR1A, ORR1B, ORR1C, and ORR1D, Long Terminal Repeats (LTRs) of MaLR endogenous retrovirus (ERV), is the direct target of Tbx6, an evolutionary conserved family of T-box TFs. Moreover, by comparing gene expression between control mice $(\mathrm{Tbx} 6+/-)$ and Tbx6-deficient mice (Tbx6 -/-), we demonstrate that at least four genes, Twist2, Pitx2, Oscp1, and Nfx/1, are down-regulated with Tbx6 deficiency. These results suggest that ORR1A, ORR1B, ORR1C and ORR1D may contribute to the evolution of mammalian embryogenesis.

Keywords: endogenous retroviruses, retrotransposon, transcription factors, evolution, TBX6

\section{INTRODUCTION}

About half of the mammalian genome is occupied by DNA sequences derived from transposable elements (TEs) (Lander et al., 2001; Waterston et al., 2002; Lindblad-Toh et al., 2005; de Koning et al., 2011). Retrotransposons, which mobilize via an RNA intermediate by a copy-and-paste mechanism, comprise the majority of mammalian TEs, whereas DNA transposons, which move via a cut-and-paste mechanism, comprise a small fraction and have accumulated mutations that render them immobile (Deininger et al., 2003). Most TEs are nonfunctional and are regarded as genomic parasites or junk DNA; however, a growing body of evidence suggests that retrotransposons and retrotransposon-derived genes have acquired functions essential for host survival during mammalian evolution (Yoder et al., 1997; Levin and Moran, 2011; Hancks and Kazazian, 2012).

In some cases, open reading frames from TEs are domesticated as endogenous genes during mammalian evolution. For example, Peg 10 and Rtll, derived from the gag and pol proteins of the Ty3/Gypsy type retrotransposon, which is similar to Sushi-ichi, are highly conserved in mammals and participate in placental formation (Ono et al., 2001, 2003, 2006; Sekita et al., 2008). Similar to the gag protein of Sushi-ichi, the other two of the eleven Sushi-ichi retrotransposon homolog (Sirh) family genes, Sirh7/Ldoc1 and Sirh11/Zcchc16, encode ORF (Open-Reading frame); they are also involved in the determination of the timing of parturition and cognitive function in the brain, respectively (Ono et al., 2011; Naruse et al., 2014; Irie et al., 2015). Syncytins/SYNCYTINs (mouse/human) and FEMATRIN (cow), derived from the envelope of endogenous retrovirus (ERV), mediate cell-cell fusion to form the syncytiotrophoblast and induce fusion with bovine endometrial cells in vitro (Mi et al., 2000; Dupressoir et al., 2009, 2011; Nakaya et al., 2013). Skin aspatic protease (SAPase), which has a retrovirus-like aspartic protease, plays important roles in the determination of the texture of the skin by modulating the degree of hydration by processing profilaggrin (Matsui et al., 2010). 
Since the discovery of TEs, it has been posited that TEs may seed regulatory elements throughout genomes and drive phenotypic differences between species via changes in transcriptional output (McClintock, 1950; Britten and Davidson, 1969; Feschotte, 2008). It has become evident that many TEs, such as long terminal repeats (LTRs) of endogenous retroviruses (ERVs), contain TF binding sites and are associated with gene expression patterns. For example, MuERV-L LTRs function as alternative promoters for protein coding genes, including Gata4 and Tead4, which are important for the specification of primitive endoderm and trophectoderm, respectively, in two-cell embryos (Kigami et al., 2003; Evsikov et al., 2004; Macfarlan et al., 2012). It has also been reported that MuERV-L, exclusively expressed in two-cell embryos, is captured at double-strand break (DSB) sites introduced by the CRISPR/Cas9 system in mouse zygotes (Ono et al., 2015). Some of the intracisterminal Aparticle (IAP) retrotransposon insertions are known to induce de novo metastable epi-alleles, such as agouti viable yellow (Avy), axin fused (AxinFu) and Cdk5rap locus (Vasicek et al., 1997; Morgan et al., 1999; Druker et al., 2004). The stochastic nature of the establishment of the epigenetic state of the $5^{\prime}$ LTR leads to variable expressivity of the adjacent genes. Both the sense and anti-sense LINE-1 (L1) promoter can drive L1 chimeric transcripts (Criscione et al., 2016). Moreover, AS071 and AS021, two AmnSINE1s present in mammals as well as birds and reptiles, are enhancers of the genes FGF8 (fibroblast growth factor 8), $178 \mathrm{~kb}$ from AS071, and SATB2, $392 \mathrm{~kb}$ from AS021 (Sasaki et al., 2008). Recently, it was reported that MER41, a primate-specific endogenized gammaretrovirus, is a source of interferon $\gamma$ (IFNG)-inducible binding sites (Chuong et al., 2016).

In this study, we demonstrate a potential role for ORR1A (Origin-Region Repeat 1A), ORR1B, ORR1C, and ORR1D, LTRs of the MaLR (Mammalian-Apparent Long-Terminal Repeat Retrotransposon) endogenous retrovirus-like element, in controlling gene expression via Tbx6 binding (Smit, 1993). Because Tbx6 functions in the regulation of early embryogenesis, including anti-neural fate regulation in the presomitic mesoderm and later somite segmentation, ORR1A, ORR1B, ORR1C, and ORR1D may have played a role in the evolution of mammalian embryogenesis (Chapman and Papaioannou, 1998; Takemoto et al., 2011).

\section{RESULTS AND DISCUSSION}

Tbx6 belongs to an evolutionarily conserved family of T-box transcription factors (TFs), known to be involved in the neuralmesodermal fate determination of axial stem cells (Chapman and Papaioannou, 1998; Takemoto et al., 2011). Previously, we revealed that Tbx6 directly activates the expression of Mesp2, a segmentation and polarization factor in somitogenesis, in a Notch signal-dependent manner (Yasuhiko et al., 2006). A ligand of Notch signal, Dll1, is also a direct target of Tbx6, implying that Tbx6 participates in the regulation of the Notch signaling pathway (White and Chapman, 2005). The consensus core sequence of Tbx6 binding sites has been reported as CACACCT or AGGTGTBRNNNN (White and Chapman, 2005). In this study, we used (C/T)CACACCT as a consensus for both reports (White and Chapman, 2005; Yasuhiko et al., 2006).

At first, the Tbx6 binding sequence motif, (C/T) CACACCT, was identified by whole genome in silico screening. Furthermore, we chose the Tbx6 binding sequence, which has at least two more Tbx6 binding sequences within the neighboring $100 \mathrm{bp}$ upstream and/or downstream regions, because we previously demonstrated that higher enhancer activity of Tbx6 was observed when there are more than three Tbx6 binding sequences within a narrow region. As a result, 3500 potential Tbx6 binding sites were identified, and a characteristic feature was revealed (Figure 1A; Supplementary Table 1).

Approximately $70.0 \%$ of potential Tbx 6 binding sites comprise repeat sequences (Figure 1A). Specifically, 85.7\% of the potential Tbx6-binding-repeat sequences were within ORR1A, ORR1B, ORR1C, and ORR1D, LTRs of the MaLR that span 679 independent ORR1s-LTRs, while SINEs and LINES represent only $2 \%$ of the Tbx6-binding repeat sequences (Bao et al., 2015; Supplementary Table 1).

There are 166,375 Repeatmasker annotated ORR1s, including partial sequences, in the mouse genome (MM10), and 20\% of them have at least one Tbx6 binding site (Figure 1B). In fact, the reference sequences of ORR1s-LTRs from Repbase, which are consensus sequences of ORR1s, have one or two Tbx6 binding sequence motifs (Figure 1C). These data suggest that the tandem insertion of these LTRs or degenerated LTR sequences with more than three Tbx6 binding sequence motifs might be good targets for Tbx6 to bind in vivo. Furthermore, potential Tbx6binding ORR1s have more than three Tbx6 binding motifs within themselves or share the Tbx6 binding motifs with neighboring sequences.

Tbx6-binding ORR1s more than 300 bp in length were selected, and the consensus sequences including three Tbx6binding motifs and the absolute distance from each ORR1 to the nearest mouse reference gene were determined (Figures 1D,E; Supplementary Table 1). The strong interaction between Tbx6 and the consensus sequence of Tbx6-binding ORR1s were confirmed by electrophoretic mobility shift assay (EMSA), while the interactions disappeared by introducing mutations into the Tbx6-binding motif one by one (Figure 1F). The finding that three Tbx6 binding motifs rather than one or two Tbx6 binding motifs have stronger binding affinity was comparable to our previous report (Figure 1G; Yasuhiko et al., 2008). As Tbx6binding ORR1s were relatively enriched near gene transcription start sites (Figure 1E), Tbx6 may contribute to regulating the expression level of nearby genes until reaching $60 \mathrm{~kb}$-windows. Then, to explore the influence of ORR1A, ORR1B, ORR1C, and ORR1D on the regulation of gene expression by $T b x 6$, we compared the expression level of 9 genes that are randomly selected within $50 \mathrm{~kb}$ of potential Tbx6 binding sites on ORR1A, ORR1B, ORR1C, and/or ORR1D in Tbx6 (+/-) (control) and Tbx6 (-/-) (Tbx6 KO) embryos at 8.0 day post-coitus (dpc). Because Tbx6 KO embryos have morphological abnormalities after $9 \mathrm{dpc}$, we used $8.0 \mathrm{dpc}$ embryos in this study to exclude secondary effects from morphological abnormalities. 
A

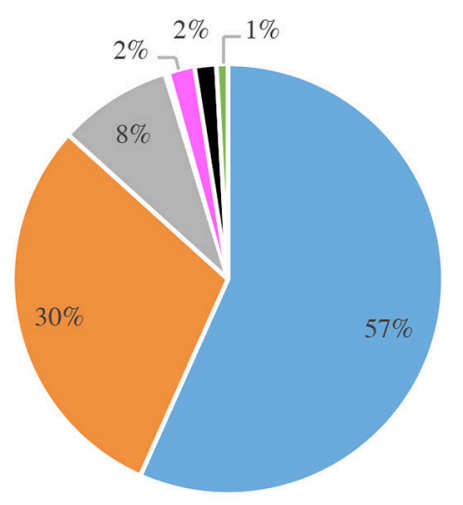

B

" ORR1s

" non-repeat

" simple repeat

"low complexity repeat

- SINE/LINE

" ERVK/ERVL

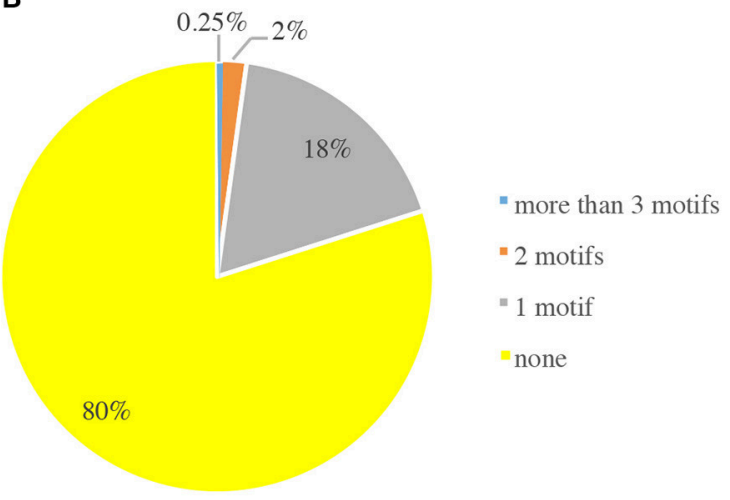

C

ORR1D

ORR1D1

ORR1A0

ORR1A2

ORR1A

ORR1A3

ORR1A4

ORR1B1

ORR1B2

ORR1B

ORR1C 1

ORR1C2

ORR1D2

ORR1E

ORR1F

ORR1G
SiteA

TTAGGAGGTGTGGCCTTGTTGGAGGA

SiteB

sitec TTAGGAGGTGTGGCCTTGTTGGAGGA-----------------AGTGTGTCA-CT--GGGGGTGGGCTTT TCTGAAGGTGTGGCCTTGTTGGAATAGGTGTGACCTGGTTGGAATGGGTGTGTCA-CTGTGGGTGTGGG-TAT TTAGGAGGTGTGGCCCTGTTGGAGTA------_---------GGTGTGTCA-CTGTGGGTGTGGGCTTT TTAGGAGGTGTGGCCTTGTTGGAGGA------------------RGTGTGTCA-CTGTGGGTGTGGGCTTT TTAGGAGGTGTGGCCTTGTTGGAGGA----------------AGTGTGTCA-CTGTGGGCGTGGGCTTT TTAGGAGGTGTGGCCTTGTTGGAGGA-----------------AGTGTGTCA-CTGTGGGGGTGGGCTTT TTAGGAGGTGTGGCCTTGTTGGAGTAGGTGTGGCCTTGTTGGAGGAAGTGTGTCA-CTGTGGGGGTGGGCTTT TTAGGAGGTGTGGCCTTGTTGGAGTAGGTGTGGCCTTGTTGGAGGAAGTGTGTCA-CT-TGGGGGTGGGCTTT TTAGGAGGTGTGGCCTTGTTGGAGGA------------------AGTGTGTCA-CT---GGGGTGGGCTTT TTAGGAGGTGTGGCCTTGTTGGAGGA----------------AGTGTGTCAACTTGGGGGGTGGGCTTT TTAGGAGGTGTGGCCTTGTTGGAGGA-----------------AGTGTGTCA-CT--GGGGGTGGGCTTT TTAGGAGGTGTGGCCTTGTTGGAGGA-----------------GGTGTGTCA-CT--GGGGGTGGGCTTT TTAGGAGGTGGAGCCTTGCTGGAGGA-----------------AGTGGGTCA-CT--GGGGGCGGGCTTT TTAAGAGGTGGGGCCTAGTAACAATGGAAG------------GAAGTTAGGTCA-TT--GGGGGTATCCCYT TTAAGAAGTGGAGCCTAGTGAGAA----------------GTYTTCAGGTCA-TT--GASAGTG-CCCTT

D

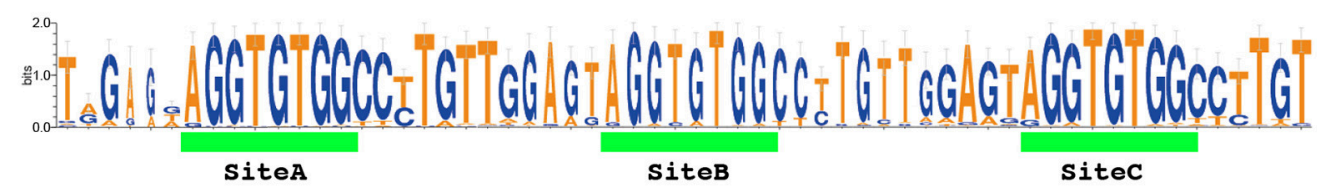

E

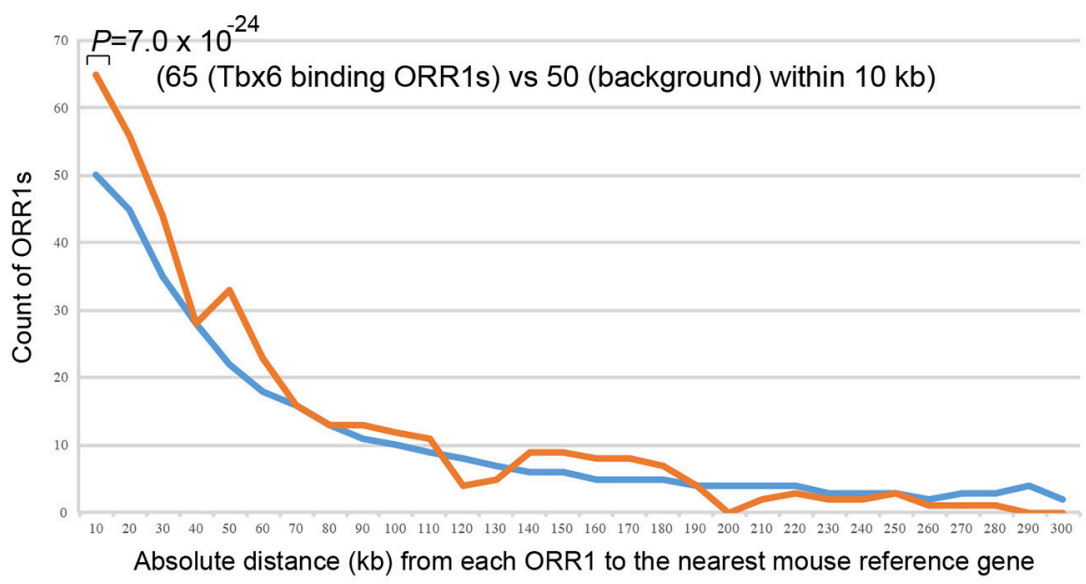

- background $\longrightarrow$ Tox 6 binding ORR1s 
$\mathbf{F}$
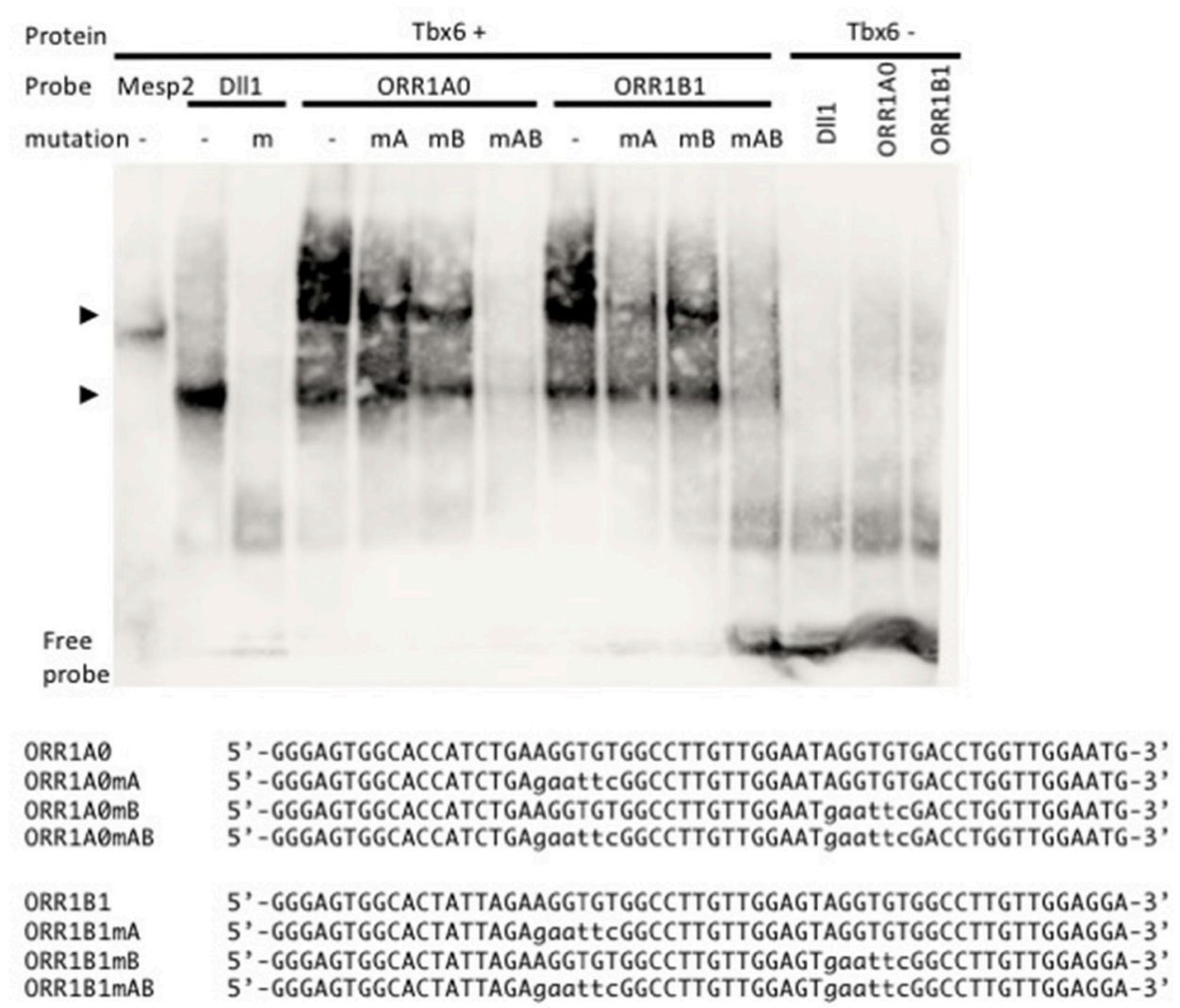

G

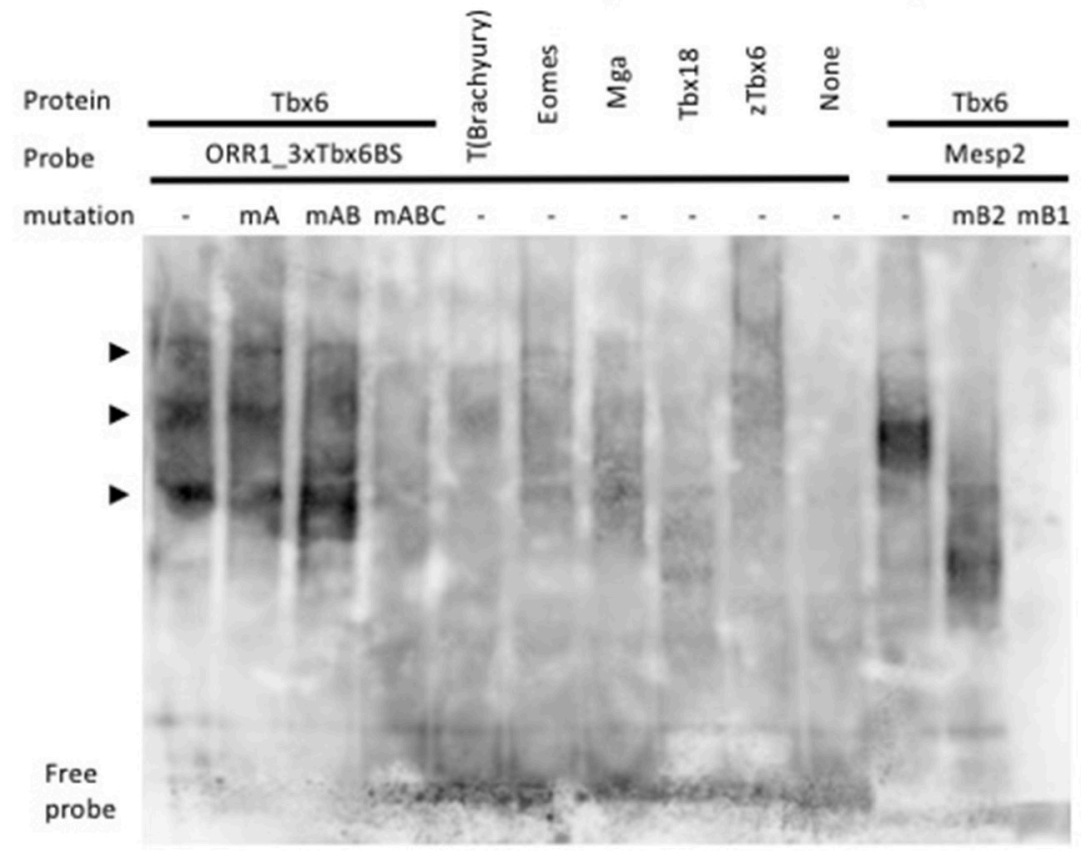

ORR1_3XTbX6BS

ORR1_3xTbx6BS $\mathrm{mA}$ ORR1_3XTbX6BS MAB ORR1_3xTbx6BS MABC
5' '-TAGAGGAGGTGTGGCCTTGTTGGAGTAGGTGTGGCCTTGTTGGAGTAGGTGTGGCCTTGT-3' 5' -TAGAGGgaattcGGCCTIGTTGGAGTAGGTGTGGCCTTGTTGGAGTAGGTGTGGCCTTGT-3' 5' -TAGAGGgaattcGGCCTIGTIGGAG gaattcGGCCTTGTTGGAGTAGGTGTGGCCTTGT-3' 5' -TAGAGGgaattcGGCCTTGTTGGAG gaattcGGCCTTGTTGGAG gaattcGGCCTTGT-3'

FIGURE 1 | Tbx6 binding sequence motifs in LTRs of ORR1 ERV families. (A) Distribution of Tbx6 binding sequence motifs in the mouse genome (MM10). Of the 3,500 Tbx6 binding sequence motifs, 70\% were repeat sequences, including TEs and simple repeats. ORR1, Long Terminal Repeats (LTRs) of MaLR endogenous 


\section{FIGURE 1 | Continued}

retrovirus (ERV), occupy $57 \%$ of the total Tbx6 binding sequence motifs. (B) Of all the ORR1 sequences in the mouse genome, $20 \%$ of ORR1s have at least one Tbx6 binding sequence. (C) DNA sequence comparison between ORR1A (rodentia ancestral shared), ORR1A0 (mus musculus), ORR1A2 (muridae), ORR1A3 (muridae), ORR1A4 (muridae), ORR1B (rodentia ancestral shared), ORR1B1 (mus musculus), ORR1B2 (mus musculus), ORR1C1 (rodentia ancestral shared), ORR1C2 (rodentia ancestral shared), ORR1D1 (rodentia ancestral shared), ORR1D2 (rodentia ancestral shared), ORR1E (rodentia ancestral shared), ORR1F (muridae) and ORR1G (muridae) LTRs. Identical sequences are indicated by asterisks. The Tbx6 binding sequence motif is indicated by green boxes. Yellow boxes are indicated as a corresponding region of the Tbx6 binding sequence motif "Site C" in Figure 1D. (D) Sequence logo of the the ORR1 LTRs that had more than three Tbx6 binding sequence motifs. Three tandem "AGGTGTGs," a Tbx6 binding sequence motif, are highly conserved between ORR1 LTRs, which have more than three Tbx6 binding sequence motifs. (E) Frequency histogram of the absolute distance from each ORR1 to the nearest mouse reference gene. The background expectation is derived from the genome-wide ORR1s distribution. Statistical significance of the observed enrichment within the first $10 \mathrm{~kb}$ of the nearest mouse reference gene was assessed by a binominal test. (F) Site A and site B sequences independently bind to Tbx6 in an electromobility shift assay (EMSA); however, the binding affinity is much higher with the presence of both sites A and B. Sequences of oligonucleotide probes were shown below the gel image. Mutated nucleotides were depicted in lower case. (G) Triple Tbx6 binding sequence motif shows the highest binding affinity to Tbx6, while other T-box TFs, including T (Brachyury), Eomes, Mga, Tbx18, and zebrafish Tbx6 (zTbx6), have no affinity. Arrowheads in (F,G): Positions of the bands resulted from multiple Tbx6 binding to ORR1 sequences. Sequences of oligonucleotide probes were shown below the gel image. Mutated nucleotides were depicted in lower case.

As expected, four genes, Twist2, Pitx2, Oscp1, and Nfxl1, were down-regulated, although the expression of five other genes, Enpep, Prdm2, Corin, Pdpn and Map4k4, was not altered significantly (Figure 2). It has been reported that enhancer activity could be blocked by the epigenetic repressive marks of the neighboring regions, such as histone deacetylation and trimethylation of $\mathrm{K} 9$ and $\mathrm{K} 27$ on histone $\mathrm{H} 3$ (H3K27me3 and $\mathrm{H} 3 \mathrm{~K} 9 \mathrm{me} 3$ ) or an insulator, a genetic boundary element blocking the interaction between enhancers and promoters (Roth et al., 2001; Schmidl et al., 2009; Greer and Shi, 2012; Dowen et al., 2014). It might be possible that five genes whose expression levels were not altered by Tbx6 deficiency could be blocked by epigenetic modifications or unknown silencers.

Our analysis revealed the rodent-specific ORR1 family of ERVs to be a source of Tbx6 binding sites. Furthermore, Tbx6-binding ORR1s are enriched near genes which might be associated with several biological process and molecular pathways (Figure 3). In the human genome, there are 2,927 potential TBX6 binding motifs; however, the majority of sites are not in LTRs but in simple repeat sequences or Alu (Supplementary Table 2). Although the source of Tbx6/TBX6 binding sequences is different between species, each mammalian species might shape their Tbx6/TBX6 binding sequence through mammalian evolution. Our analysis and other reports, including the primate-specific MER41 family as IFNG-inducible binding sites and AmnSINE1s as mammalian enhancers, raised the possibility that TE-derived regulatory elements influence lineagespecific mammalian evolution (Sasaki et al., 2008; Chuong et al., 2016).

\section{MATERIALS AND METHODS}

\section{Bioinformatic Analyses}

(C/T)CACACCT, Tbx6 binding sequence motifs, were identified in the mouse whole genome (MM10) and human whole genome (hg19) and filtered out when there were not two more Tbx6 binding sequences within the neighboring $100 \mathrm{bp}$ upstream and/or downstream regions using gggenome (https:// gggenome.dbcls.jp). All the TE sequences were downloaded from Repeatmasker truck (mouse:MM10/human:hg19) of the UCSC genome browser (https://genome.ucsc.edu). The
Intersect intervals program (https://usegalaxy.org/) was used to identify the TEs that have potential Tbx6 binding sequences using potential Tbx6 binding sites identified as a query against Repeatmasker annotated TEs. The ClosestBed program (https:// usegalaxy.org/) was used to find the closest mouse reference genes (MM10) and to identify the absolute distance between the potential Tbx6 binding motif and its closest reference gene. These distances were grouped by $10 \mathrm{~kb}$-bin sizes. The expected background was determined by randomly sampling an equal number of the remaining 78,042 annotated ORR1s that did not have more than three Tbx binding motifs. Sampling was repeated 100 times, and the mean number of elements was used as the expected value for comparison to the potential Tbx6 binding ORR1s. Statistical significance was determined for the first $10-\mathrm{kb}$ bin by a binominal test as previously described (Chuong et al., 2016). Gene ontology of the closest reference genes within $50 \mathrm{~kb}$-windows of potential Tbx6-binding motifs were determined by the GREAT program http://bejerano. stanford.edu/great/public/html/index.php (Figure 3). The consensus sequence of the potential Tbx6-binding motifs was identified by ClustalW program (for alignment: http://clustalw. ddbj.nig.ac.jp/index.php?lang=ja)and Sequence Logo program (for generation of sequence logos: http://weblogo.berkeley. edu). A gene ontology/signal pathway panel of Tbx6-binding ORR1s.

\section{Electrophoretic Mobility Shift Assay (EMSA)}

Full sequences of ORFs of mouse Tbx6 (NM_011538.2), $T$ (Brachyury; NM_009309.2), Eomes (NM_010136.3), Tbx18 (NM_023814.4), and T-box-coding fragment of Mga (NM_013720.2) were PCR amplified and cloned in pCS2+ (Rupp et al., 1994) vector. Expression vector pCS2-zTbx6 for zebrafish Tbx6 translation was a gift from Dr Hiroyuki Takeda (Terasaki et al., 2006). Transcription factors were in vitro transcribed and translated using TnT(R) Quick Coupled Transcription/Translation System (Promega) following the manufacturer's protocol. Sequences of DNA probes were as follows: Mutated nucleotides are designated in lower case. Mesp2 and Dll1 were positive controls for the assay and described in Yasuhiko et al. (2006) and 


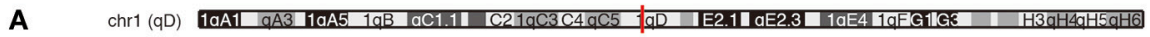

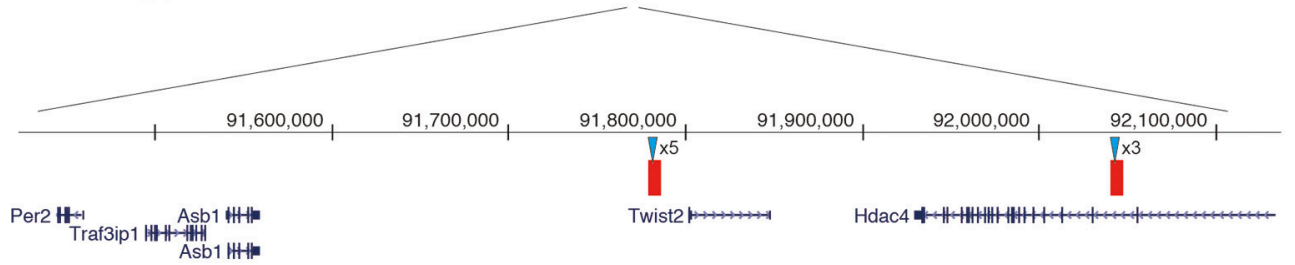

Twist2

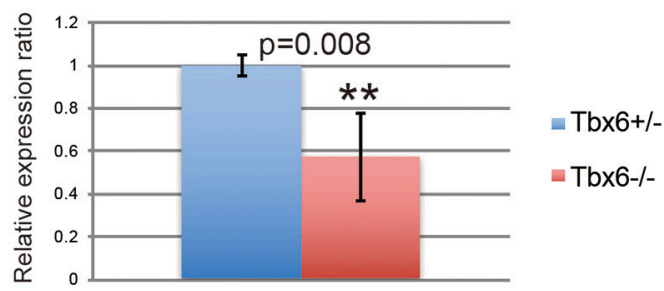
chr1: I
$91,787,000$ I
$91,788,0001$
$91,789,0001$
$91,790,000$ ।
$91,791,000$
$91,792,000$ I ORR1C2\#LTR/ERVL-MaLR «ere «e

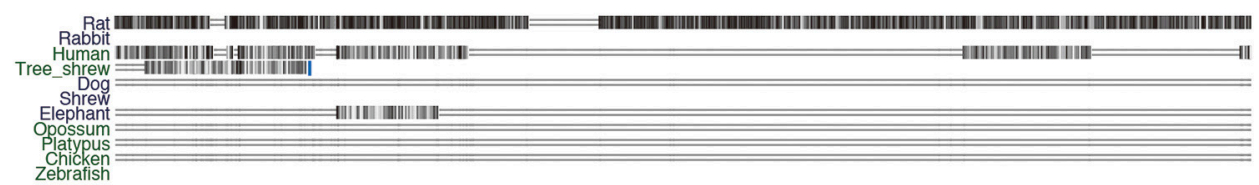

B

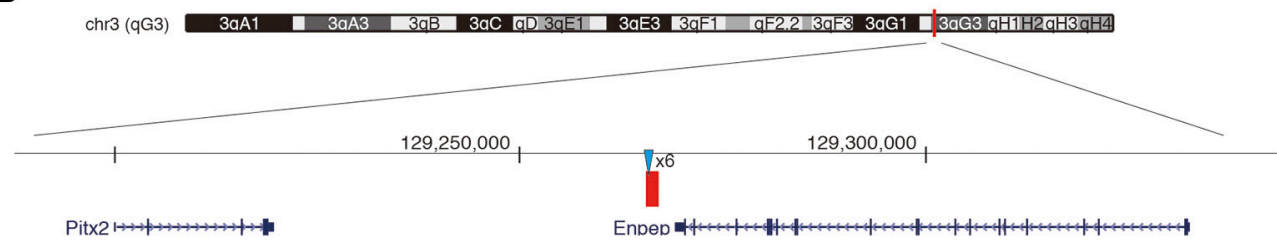

Pitx2

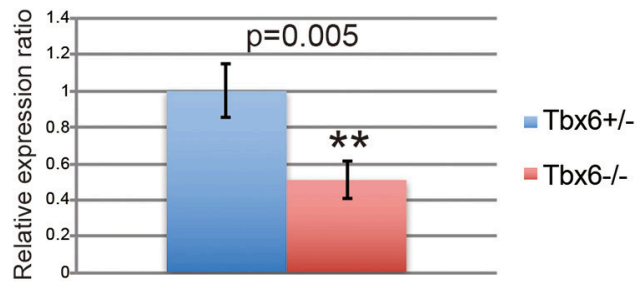

Enpep

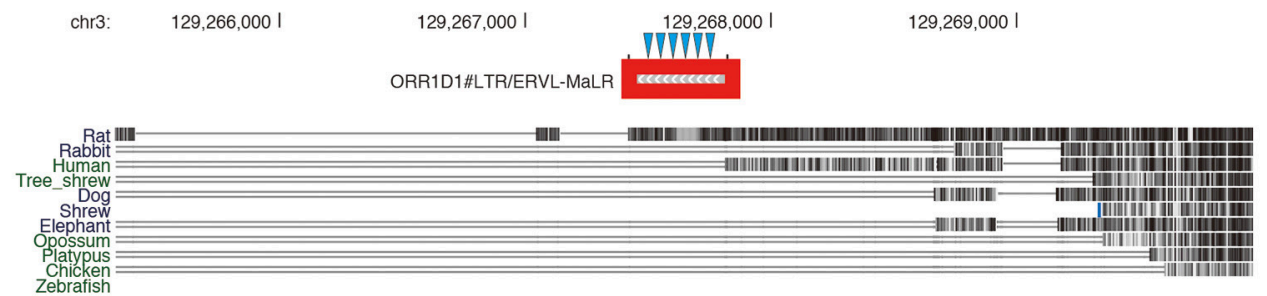




\section{C}
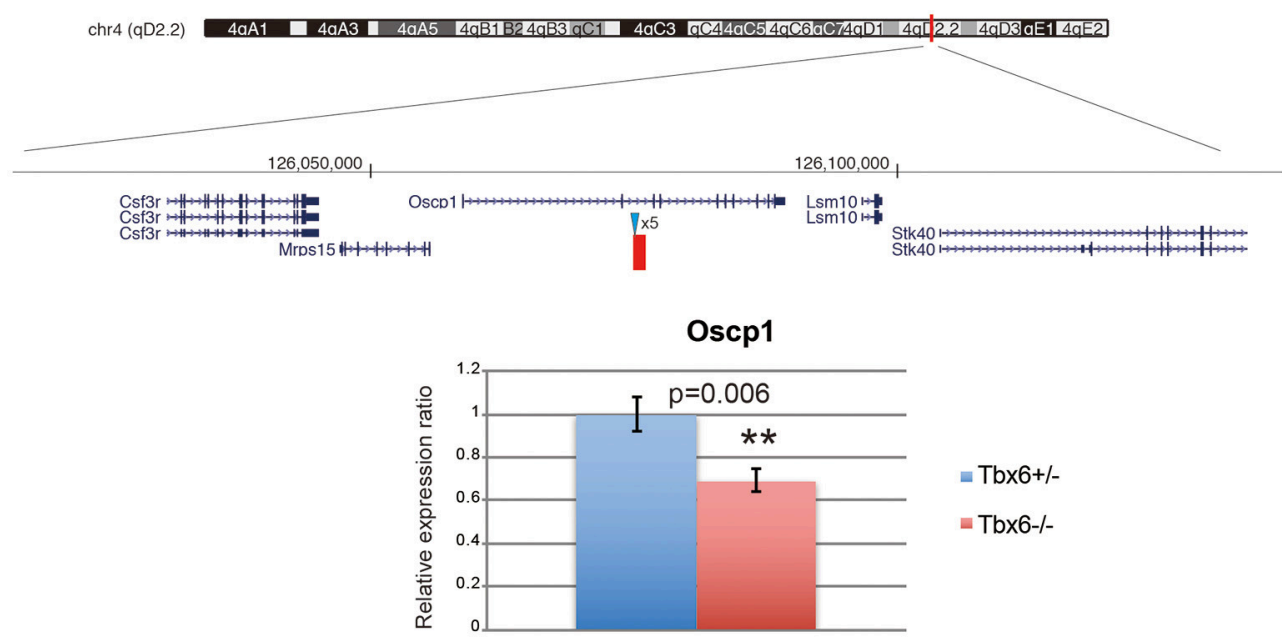

$=\mathrm{Tbx} 6+/-$

- Tbx6-/-

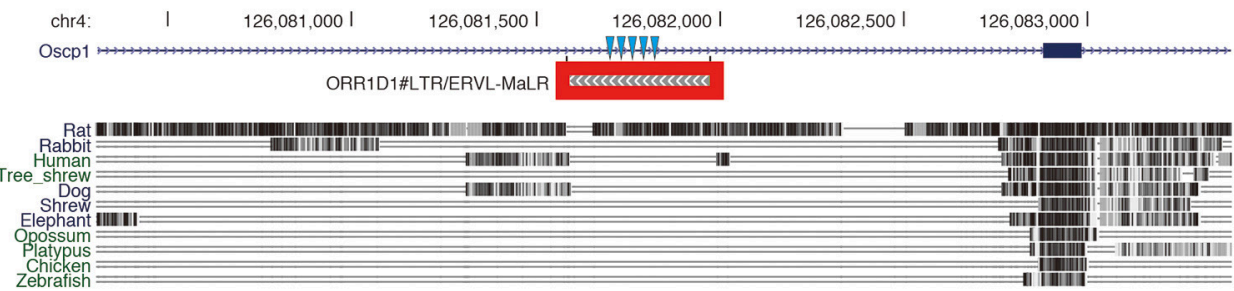

D

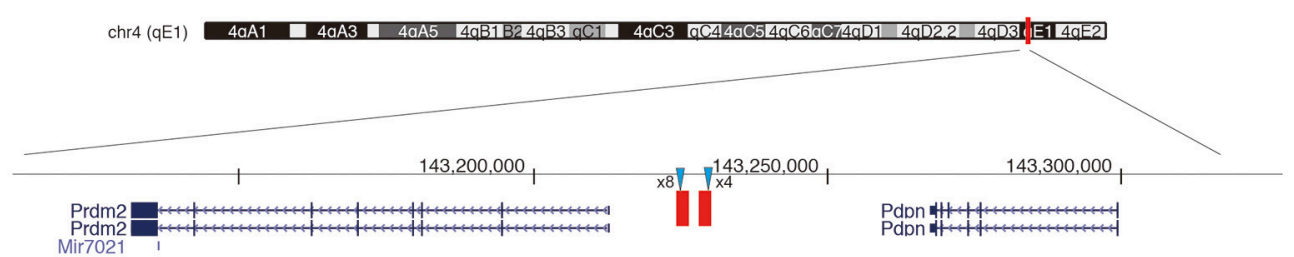

Prdm2

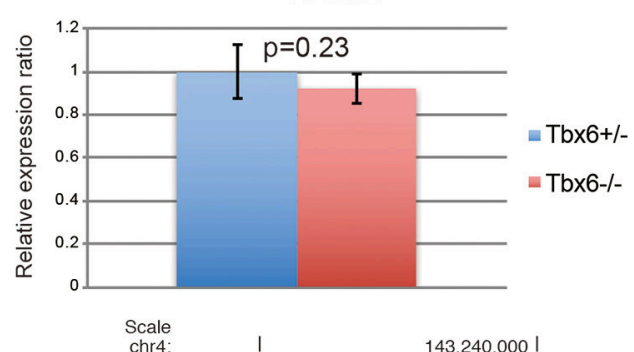

IVTYWN

ORR1A2\#LTR/ERVL-MaLR IKI

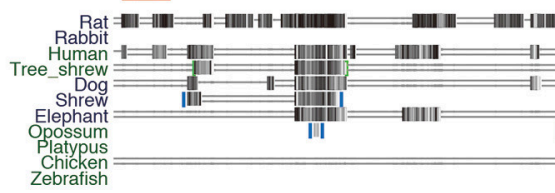
ORRIALHLTR
Pdpn

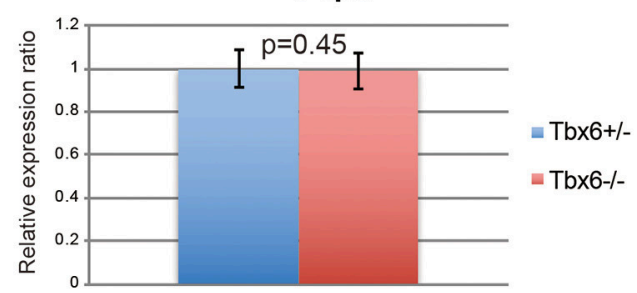

$143,245,0001$

$143,250,000$ I

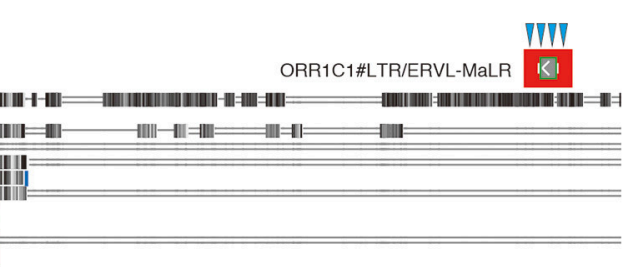


E

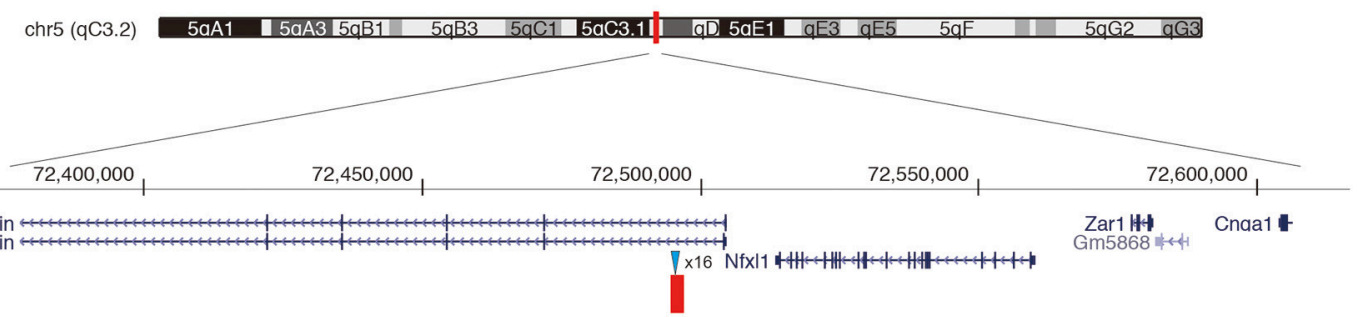

\section{Corin}

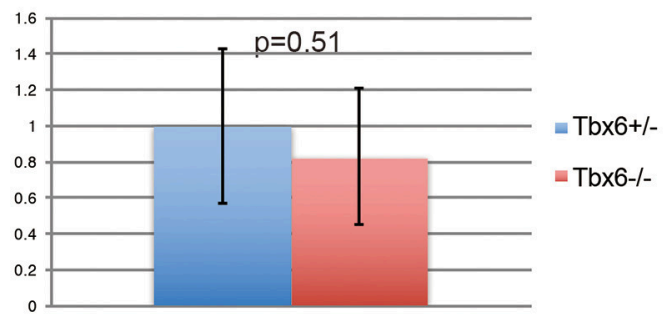

Nfxl1

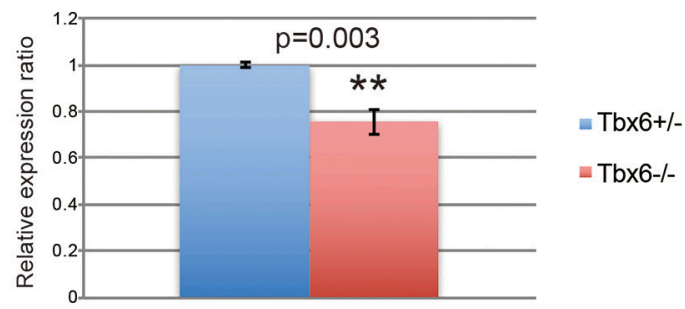

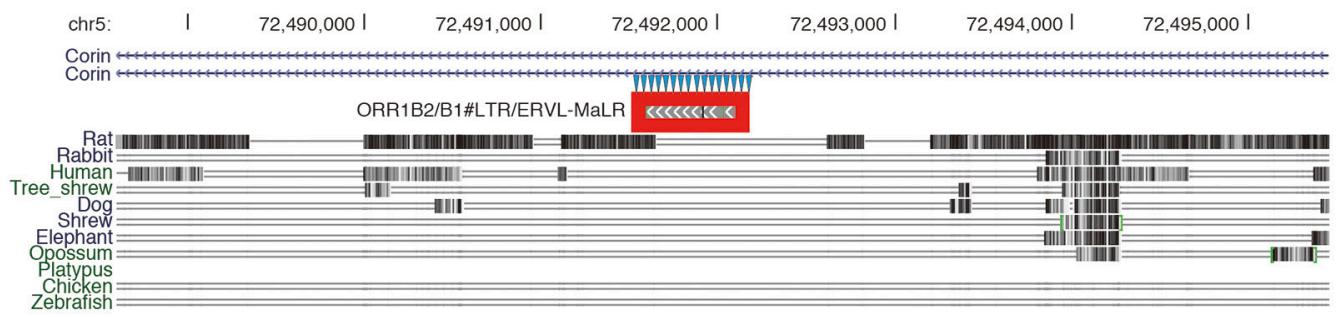

$\mathbf{F}$
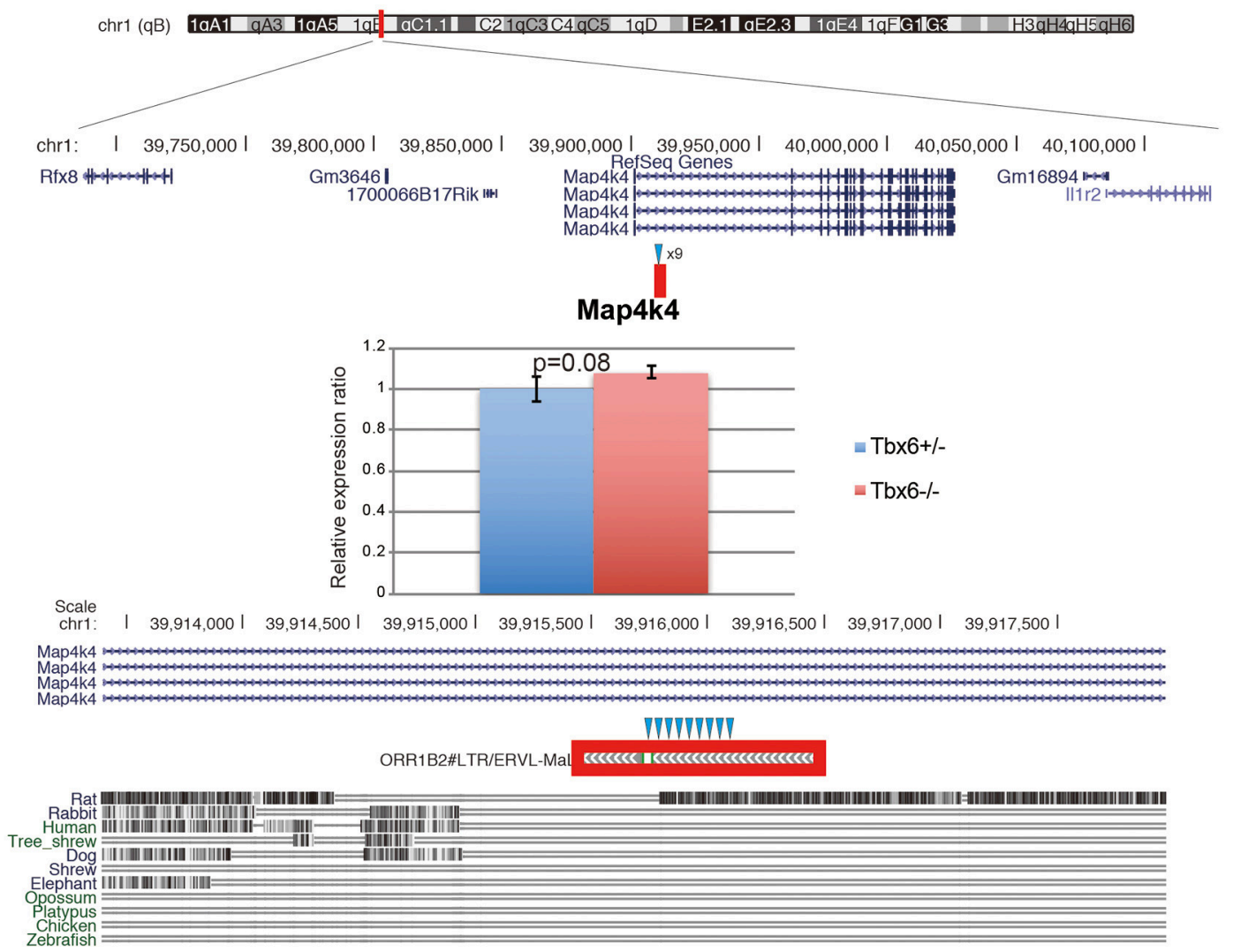

FIGURE 2 | Continued 
G
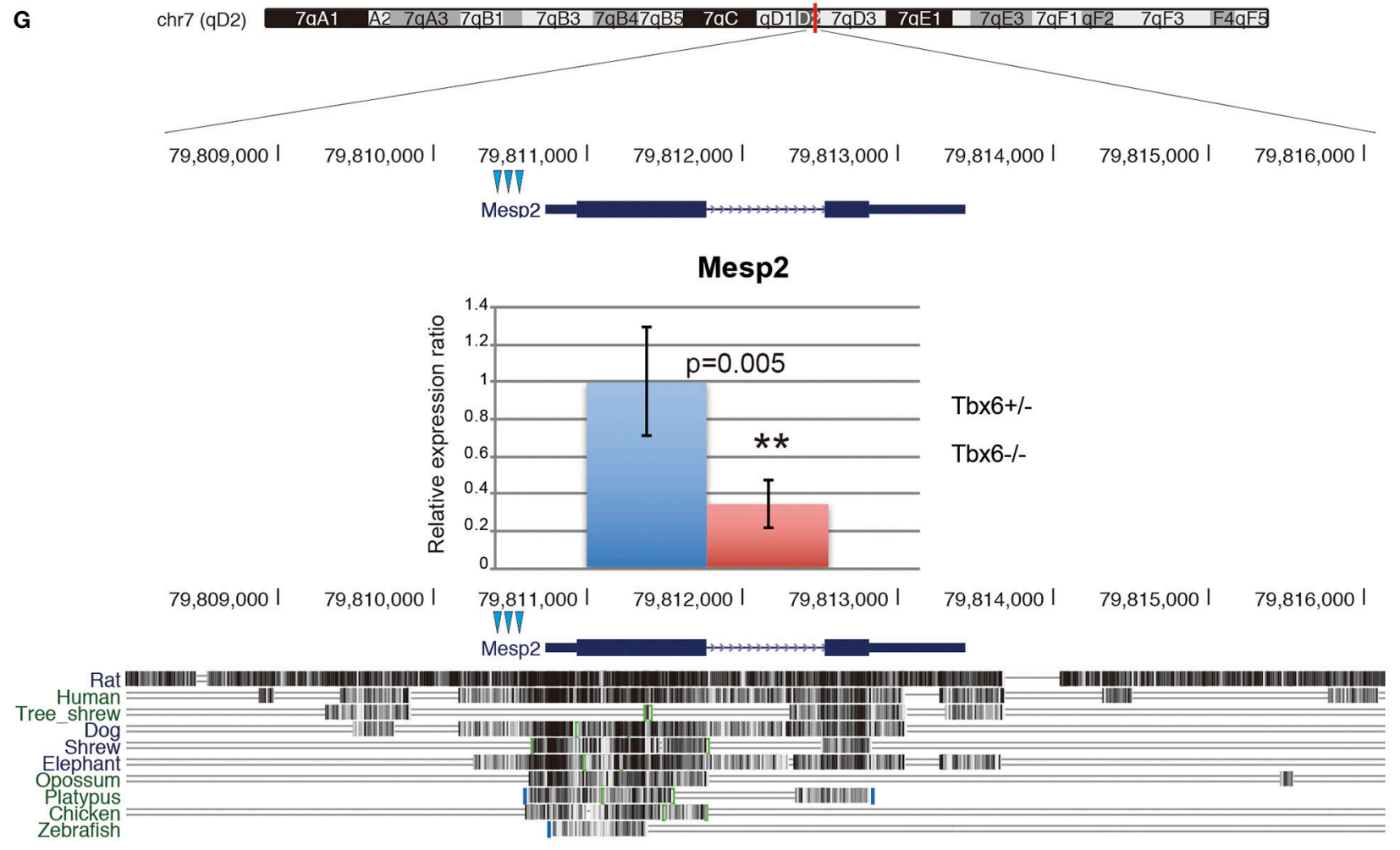

H

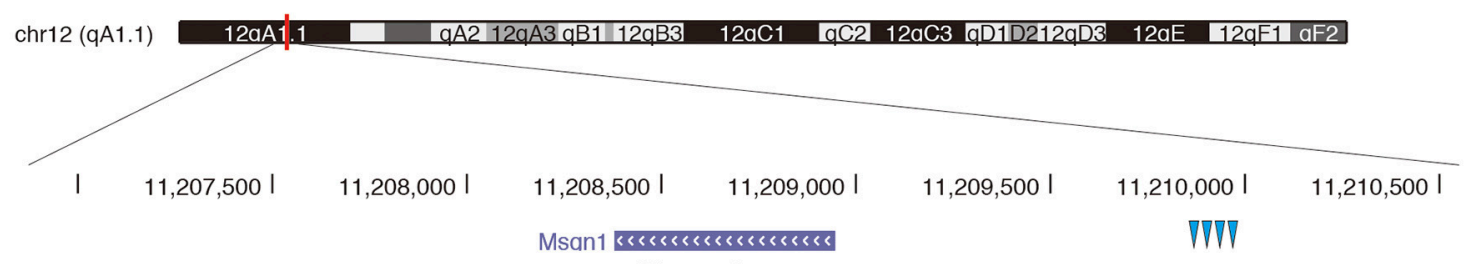

Msgn1

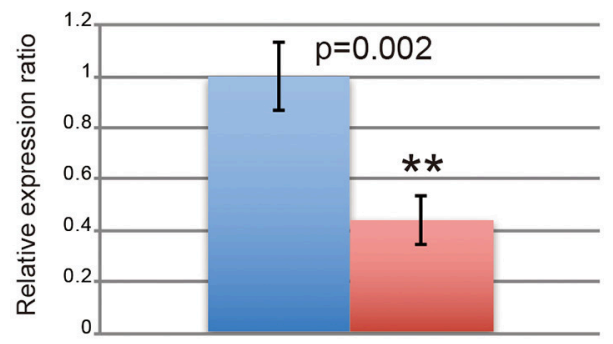

Tbx6+/-

Tbx6-/-

Msan1 reresersersereses

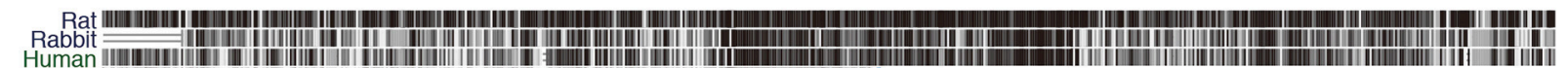
Tree shrew

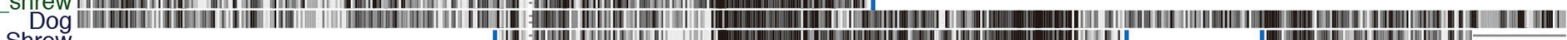

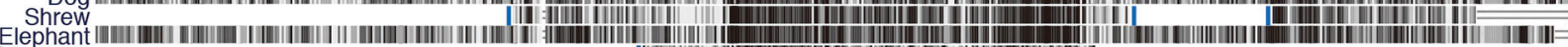
Elephant

Platypus

chicken

|

||

FIGURE 2 | Continued 

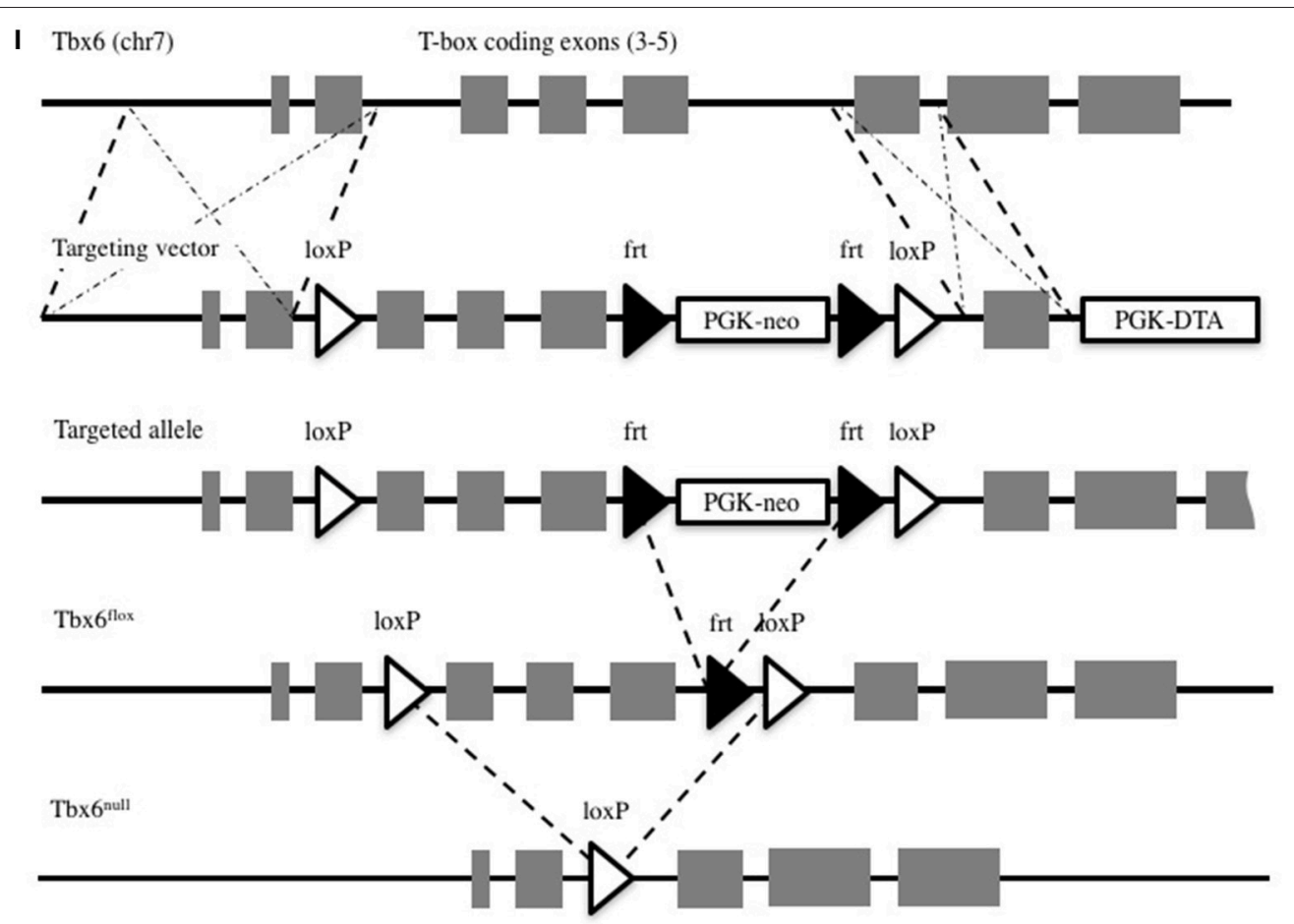

FIGURE 2 | ORR1 family of ERVs function as enhancers by Tbx6-binding. Positions of LTRs including potential Tbx6 binding sites (red bars) are indicated in the upper side of each panel (A-F). Relative expression levels were determined by quantitative RT-PCR of the Tbx6-binding-LTRs adjacent genes Twist2 (A), Pitx2 (B), Enpep (B), Oscp1 (C), Prdm2 (D), Pdpn (D), Nfxl1 (E), and Map4k4 (F) and the previously reported positive control genes Mesp2 (G) and Msgn1 (H) in Tbx6 (+/-) (control: blue bars) and Tbx6 (-/-) (Tbx6 KO: red bars) embryos at $8.0 \mathrm{dpc}$. The relative expression ratios are normalized to the housekeeping gene $\beta$-actin. The details of Tbx6-binding LTRs and conservation among vertebrate species. The Tbx6-binding LTRs (red boxes) are also conserved in rats (bottom of A-C); however, another three are not conserved in other rodents (bottom of $\mathbf{D}-\mathbf{F})$. Tbx6-binding motifs are indicated by blue triangles. Schematic description of Tbx6 conditional KO mouse was shown in (I).

White and Chapman (2005), respectively. 1ORR1A0, 5' GGGAGTGGCACCATCTGAAGGTGTGGCCTTGTTGGAATA GGTGTGACCTGGTTGGAATG-3'; ORR1A0mA, 5'-GGGAG TGGCACCATCTGAgaattcGGCCTTGTTGGAATAGGTGTGA CCTGGTTGGAATG-3'; ORR1A0mB， 5'-GGGAGTGGCAC CATCTGAAGGTGTGGCCTTGTTGGAATgaattcGACCTGG TTGGAATG-3'; ORR1A0mAB, 5'-GGGAGTGGCACCATCTG AgaattcGGCCTTGTTGGAATgaattcGACCTGGTTGGAATG3'; ORR1B1, 5'-GGGAGTGGCACTATTAGAAGGTGTGG CCTTGTTGGAGTAGGTGTGGCCTTGTTGGAGGA-3'; ORR1B1mA, 5'-GGGAGTGGCACTATTAGAgaattcGGC CTTGTTGGAGTAGGTGTGGCCTTGTTGGAGGA-3';

ORR1B1mB, 5'-GGGAGTGGCACTATTAGAAGGTGT GGCCTTGTTGGAGTgaattcGGCCTTGTTGGAGGA-3';

ORR1B1mAB, 5'-GGGAGTGGCACTATTAGAgaattcG GCCTTGTTGGAGTgaattcGGCCTTGTTGGAGGA-3';

ORR1_3xTbx6BS, 5'-TAGAGGAGGTGTGGCCTTGTTG GAGTAGGTGTGGCCTTGTTGGAGTAGGTGTGGCCTTG

T-3'; ORR1_3xTbx6BSmA, 5'-TAGAGGgaattcGGCCTTG TTGGAGTAGGTGTGGCCTTGTTGGAGTAGGTGTGGCCT TGT-3'; ORR1_3xTbx6BSmAB, 5'-TAGAGGgaattcGGCCT TGTTGGAGTgaattcGGCCTTGTTGGAGTAGGTGTGGCC TTGT-3'; ORR1_3xTbx6BSmABC， 5'-TAGAGGgaattcGGCC TTGTTGGAGTgaattcGGCCTTGTTGGAGTgaattcGGCCTTG
T-3'; Mesp2, 5'-CCTTCGAGGGGTCAGAATCCACACCTC TGCAAATGGGCCCGCTTT-3'; Mesp2mB2, 5' - CCTTCG AGaGtaCtGAATCCACACCTCTGCAAATGGGCCCGCTTT3'; Mesp2mB1, 5' - CCTTCGAGGGGTCAGAATCgAtAtCTCT GCAAATGGGCCCGCTTT-3'; Dll1， 5'-ACAATCAAAGGA ACACTAGCTCCAAGAATCACACCTCGGGATTCTAATG AAGCTGCCTA-3'; Dll1m, 5' -ACAATCAAAGGAACACTA GCTCCAAGAATCgaattcCGGGATTCTAATGAAGCTGCC

TA-3'. Sense and anti-sense oligonucleotides for each probe were annealed, DIG-labeled and subjected to EMSA assay using the DIG Gel Shift Kit, 2nd Generation (Roche). Briefly, 4 fmol of labeled oligonucleotide probe was incubated with $4 \mu \mathrm{l}$ of in vitro translated mixture, electrophoresed in $7.5 \%$ polyacrylamide gel for $80 \mathrm{~min}$ at $100 \mathrm{~V}$, and blotted to a positively charged nylon membrane. Shifted oligonucleotides were detected using an anti-DIG Fab fragment (Roche) and CDP-Star Ready-to-Use AP substrate (Roche).

\section{Gene Targeting, Mouse Embryos and Real-Time RT-PCR}

All animal studies were conducted in accordance with the guidelines approved by the animal care committee of the National Institute of Health Sciences (NIHS; No.934). The 


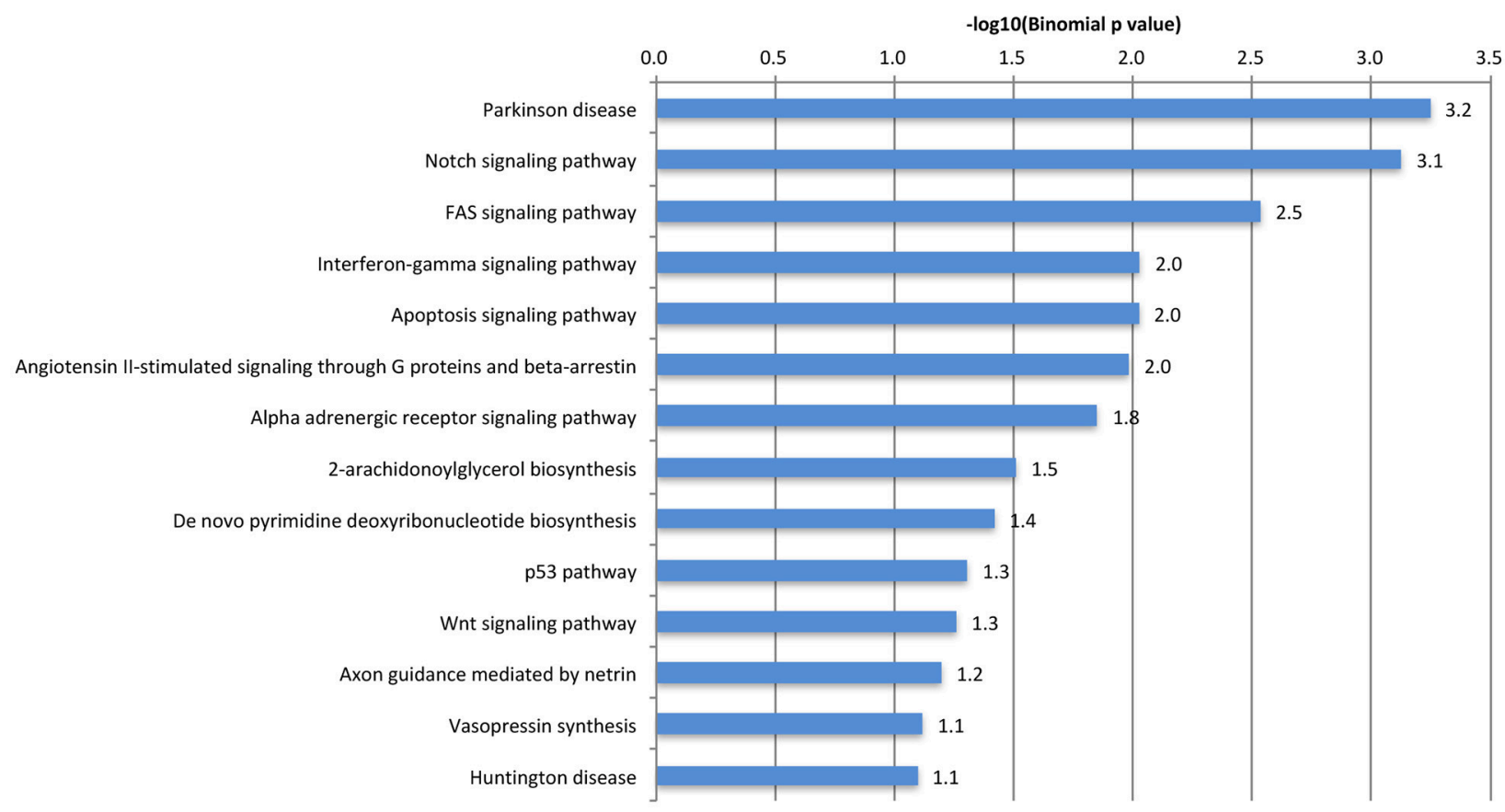

B

\section{GO Molecular Function}

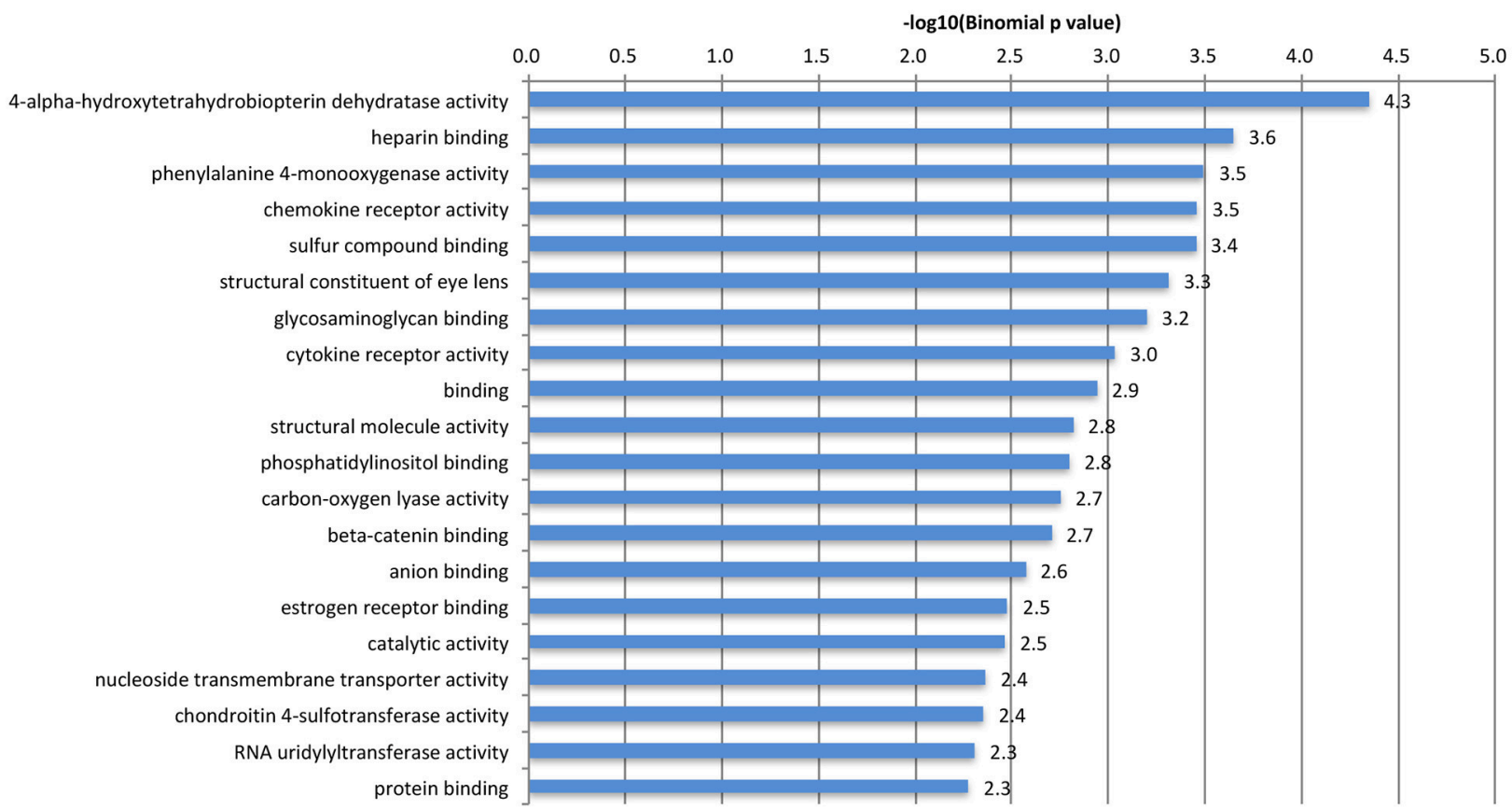

FIGURE 3 | A gene ontology/signal pathway panel of Tbx6-binding ORR1s. Top categories of GO Biological Process were shown. (A) Top categories of Signal Pathway were shown. (B) All displayed categories were significant by binomial test $(P<0.1)$. 
protocol was approved by the animal welfare committee of National Institute of Health Sciences (NIHS; No.41). Animals had access to a standard chow diet and water ad libitum and were housed in a pathogen-free barrier facility with a 12L:12D cycle.

A Tbx6 conditional knockout mouse (Tbx6flox) was generated using the ES cell line TT2 and maintained in an ICR background (Yagi et al., 1993). Briefly, exon 3-5, encoding the T-box DNA binding domain of Tbx6, was flanked by a pair of loxP sites and knocked into the Tbx6 locus by homologous recombination. The PGK-neo selection marker was removed by the FLP-FRT system to obtain Tbx6flox mice. For cDNA preparation, embryos (8.0 days post-coitus) were obtained by crossing female CAG-Cre/Tbx6flox/+ hybrid heterozygotes onto male Tbx6flox/flox homozygotes. Embryos were genotyped by PCR using allantois genomic DNA, and total RNA were prepared using an RNeasy mini kit (QIAGEN). Total RNA was pooled from 5 (Tbx6+/-) and 4 (Tbx6-/-) 8.0dpc sibling embryos in the same litter. The sequences of primers for real-time RT-PCR were as follows:

Twist2_forward, 5' -TGTCCGCCTCCCACTAGC-3';

Twist2_reverse, 5' -TGTCCAGGTGCCGAAAGTC-3';

Pitx2_forward, 5'-GGCAGTCACCCTGGGAAG-3';

Pitx2_reverse, 5'-GCCGACACTAGTTTGCGACA-3';

Enpep_forward, 5'-CCTGCTTTACGACCCCCTAC-3';

Enpep_reverse, 5' -TTAGCCACAAGTCGTCCCAC-3';

Oscp1_forward, 5' -GACTCTGCCGCTGCTCT-3';

Oscp1_reverse, 5' -TCGTCCATGAACTTCCTGTTGA-3';

Prdm2_forward, 5'-GCTTCGAGGACTTCCAGAGG-3';

Prdm2_reverse, 5' -TGGTTTAGTGGCCCAGACAC-3';

$P d p n$ forward, $5^{\prime}$-AGGTGCTACTGGAGGGCTTA-3';

$P d p n \_$reverse, $5^{\prime}$-GCTGAGGTGGACAGTTCCTC-3';

Nfxl1_forward, 5' -AGAACCTCCTCAGTTGCTGC-3';

Nfxl1_reverse, 5'-AAGGGGCATTCACCAGGATG-3';

Corin_forward, $5^{\prime}$-GATATGTTCACGAAACGGCCC-3';

Corin_reverse, 5' -CGCTCCTGTCTGCTCTCAAG-3';

Map4k4_forward, 5' -TTCCGGCCTCTCAAGCCT-3';

Map4k4_reverse, 5' -TCCCAGACTCCTCACTGGAG-3';

Mesp2_forward, 5' -ACCCTTACACCAGTCCCTAGAAA-3';

Mesp2_reverse, 5' -GGTTCTGGAGACACAGAAAGACT-3';

$M s g n 1$ forward, 5' -GCCAGAAAGGCAGCAAAGTC- $3^{\prime}$;

Msgn1_reverse, 5' -AGACAGGCGGCAGGTAATTC-3';

$\beta$-actin_forward, 5' -CTGTCGAGTCGCGTCCA-3';

$\beta$-actin_reverse, $5^{\prime}$-ACGATGGAGGGGAATACAGC-3';

\section{REFERENCES}

Bao, W., Kojima, K. K., and Kohany, O. (2015). Repbase Update, a database of repetitive elements in eukaryotic genomes. Mob. DNA 6, 11. doi: 10.1186/s13100-015-0041-9

Britten, R. J., and Davidson, E. H. (1969). Gene regulation for higher cells: a theory. Science 165, 349-357. doi: 10.1126/science.165.3891.349

Chapman, D. L., and Papaioannou, V. E. (1998). Three neural tubes in mouse embryos with mutations in the T-box gene Tbx6. Nature 391, 695-697.

Chuong, E. B., Elde, N. C., and Feschotte, C. (2016). Regulatory evolution of innate immunity through co-option of endogenous retroviruses. Science 351, 1083-1087. doi: 10.1126/science.aad5497

Criscione, S. W., Theodosakis, N., Micevic, G., Cornish, T. C., Burns, K. H., Neretti, N., et al. (2016). Genome-wide characterization of
Primers were designed using Primer-BLAST (https://www. ncbi.nlm.nih.gov/tools/primer-blast/) tool to amplify 70-150 base pair (bp) fragment separated by at least one intron (>500 bp), except Msgn1 (single exon gene). PCR reaction was performed using SYBR(R) Premix Ex Taq(TM) II (Takara RR820S) following the manufacturer's protocol, with PCR cycle as follows: 1 cycle of $95^{\circ} \mathrm{C} 30 \mathrm{~s}, 40$ cycles of $95^{\circ} \mathrm{C} 5 \mathrm{~s}$ and $60^{\circ} \mathrm{C} 30 \mathrm{~s}$.

\section{Statistical Analyses}

Statistical significance for qPCR was assessed using a two-tailed unpaired Student's $t$-test with a threshold of $p<0.1$.

\section{ETHICS STATEMENT}

The animal facility of the National Institute of Health Sciences was approved by the Japan Health Sciences Foundation since 2008. All animal studies were conducted in accordance with the guidelines approved by the animal welfare committee of the National Institute of Health Sciences (NIHS; No. 41).

\section{AUTHOR CONTRIBUTIONS}

$\mathrm{RO}$ conceived of the project. YY, YH, and RO participated in the experimental design. RO performed most analyses. YY produced Tbx6 KO mice and performed EMSA and RT-PCR. RO wrote the manuscript. All authors read and approved the final manuscript.

\section{ACKNOWLEDGMENTS}

This work was supported by grants from Grant-in-Aid for Scientific Research (C) (26430183) and the Research on Regulatory Science of Pharmaceuticals and Medical Devices from Japan Agency for Medical Research and Development, AMED to RO. We are grateful to Hiroyuki Takeda (University of Tokyo) for providing $z$ Tbx 6 cDNA clones, and to Satoshi Kitajima and Eriko Ikeno for technical assistance in generating Tbx6 KO mouse.

\section{SUPPLEMENTARY MATERIAL}

The Supplementary Material for this article can be found online at: http://journal.frontiersin.org/article/10.3389/fchem. 2017.00034/full\#supplementary-material

human L1 antisense promoter-driven transcripts. BMC Genomics 17:463. doi: 10.1186/s12864-016-2800-5

Deininger, P. L., Moran, J. V., Batzer, M. A., and Kazazian, H. H. Jr. (2003). Mobile elements and mammalian genome evolution. Curr. Opin. Genet. Dev. 13, 651-658. doi: 10.1016/j.gde.2003.10.013

de Koning, A. P., Gu, W., Castoe, T. A., Batzer, M. A., and Pollock, D. D. (2011). Repetitive elements may comprise over two-thirds of the human genome. PLoS Genet. 7:e1002384. doi: 10.1371/journal.pgen.1002384

Dowen, J. M., Fan, Z. P., Hnisz, D., Ren, G., Abraham, B. J., Zhang, L. N., et al. (2014). Control of cell identity genes occurs in insulated neighborhoods in mammalian chromosomes. Cell 159, 374-387. doi: 10.1016/j.cell.2014.09.030

Druker, R., Bruxner, T. J., Lehrbach, N. J., and Whitelaw, E. (2004). Complex patterns of transcription at the insertion site of a retrotransposon in the mouse. Nucleic Acids Res. 32, 5800-5808. doi: 10.1093/nar/gkh914 
Dupressoir, A., Vernochet, C., Bawa, O., Harper, F., Pierron, G., Opolon, P., et al. (2009). Syncytin-A knockout mice demonstrate the critical role in placentation of a fusogenic, endogenous retrovirus-derived, envelope gene. Proc. Natl. Acad. Sci. U.S.A. 106, 12127-12132. doi: 10.1073/pnas.0902925106

Dupressoir, A., Vernochet, C., Harper, F., Guegan, J., Dessen, P., Pierron, G., et al. (2011). A pair of co-opted retroviral envelope syncytin genes is required for formation of the two-layered murine placental syncytiotrophoblast. Proc. Natl. Acad. Sci. U.S.A. 108, E1164-E1173. doi: 10.1073/pnas.1112304108

Evsikov, A. V., de Vries, W. N., Peaston, A. E., Radford, E. E., Fancher, K. S., Chen, F. H., et al. (2004). Systems biology of the 2-cell mouse embryo. Cytogenet. Genome Res. 105, 240-250. doi: 10.1159/000078195

Feschotte, C. (2008). Transposable elements and the evolution of regulatory networks. Nat. Rev. Genet. 9, 397-405. doi: 10.1038/nrg2337

Greer, E. L., and Shi, Y. (2012). Histone methylation: a dynamic mark in health, disease and inheritance. Nat. Rev. Genet. 13, 343-357. doi: 10.1038/nrg3173

Hancks, D. C., and Kazazian, H. H. Jr. (2012). Active human retrotransposons: variation and disease. Curr. Opin. Genet. Dev. 22, 191-203. doi: 10.1016/j.gde.2012.02.006

Irie, M., Yoshikawa, M., Ono, R., Iwafune, H., Furuse, T., Yamada, I., et al. (2015). Cognitive Function Related to the Sirh11/Zcchc16 Gene Acquired from an LTR Retrotransposon in Eutherians. PLoS Genet. 11:e1005521. doi: 10.1371/journal.pgen.1005521

Kigami, D., Minami, N., Takayama, H., and Imai, H. (2003). MuERV-L is one of the earliest transcribed genes in mouse one-cell embryos. Biol. Reprod. 68, 651-654. doi: 10.1095/biolreprod.102.007906

Lander, E. S., Linton, L. M., Birren, B., Nusbaum, C., Zody, M. C., Baldwin, J., et al. (2001). Initial sequencing and analysis of the human genome. Nature 409, 860-921. doi: 10.1038/35057062

Levin, H. L., and Moran, J. V. (2011). Dynamic interactions between transposable elements and their hosts. Nat. Rev. Genet. 12, 615-627. doi: 10.1038/nrg3030

Lindblad-Toh, K., Wade, C. M., Mikkelsen, T. S., Karlsson, E. K., Jaffe, D. B., Kamal, M., et al. (2005). Genome sequence, comparative analysis and haplotype structure of the domestic dog. Nature 438, 803-819. doi: 10.1038/nature04338

Macfarlan, T. S., Gifford, W. D., Driscoll, S., Lettieri, K., Rowe, H. M., Bonanomi, D., et al. (2012). Embryonic stem cell potency fluctuates with endogenous retrovirus activity. Nature 487, 57-63. doi: 10.1038/nature11244

Matsui, T., Leung, D., Miyashita, H., Maksakova, I. A., Miyachi, H., Kimura, H., et al. (2010). Proviral silencing in embryonic stem cells requires the histone methyltransferase ESET. Nature 464, 927-931. doi: 10.1038/nature08858

McClintock, B. (1950). The origin and behavior of mutable loci in maize. Proc. Natl. Acad. Sci. U.S.A. 36, 344-355. doi: 10.1073/pnas.36.6.344

Mi, S., Lee, X., Li, X., Veldman, G. M., Finnerty, H., Racie, L., et al. (2000). Syncytin is a captive retroviral envelope protein involved in human placental morphogenesis. Nature 403, 785-789. doi: 10.1038/35001608

Morgan, H. D., Sutherland, H. G., Martin, D. I., and Whitelaw, E. (1999). Epigenetic inheritance at the agouti locus in the mouse. Nat. Genet. 23, 314-318. doi: $10.1038 / 15490$

Nakaya, Y., Koshi, K., Nakagawa, S., Hashizume, K., and Miyazawa, T. (2013). Fematrin-1 is involved in fetomaternal cell-to-cell fusion in Bovinae placenta and has contributed to diversity of ruminant placentation. J. Virol. 87, 10563-10572. doi: 10.1128/JVI.01398-13

Naruse, M., Ono, R., Irie, M., Nakamura, K., Furuse, T., Hino, T., et al. (2014). Sirh7/Ldoc1 knockout mice exhibit placental P4 overproduction and delayed parturition. Development 141, 4763-4771. doi: 10.1242/dev.114520

Ono, R., Ishii, M., Fujihara, Y., Kitazawa, M., Usami, T., Kaneko-Ishino, T., et al. (2015). Double strand break repair by capture of retrotransposon sequences and reverse-transcribed spliced mRNA sequences in mouse zygotes. Sci. Rep. 5:12281. doi: 10.1038/srep12281

Ono, R., Kobayashi, S., Wagatsuma, H., Aisaka, K., Kohda, T., KanekoIshino, T., et al. (2001). A retrotransposon-derived gene, PEG10, is a novel imprinted gene located on human chromosome 7q21. Genomics 73, 232-237. doi: 10.1006/geno.2001.6494

Ono, R., Kuroki, Y., Naruse, M., Ishii, M., Iwasaki, S., Toyoda, A., et al. (2011). Identification of tammar wallaby SIRH12, derived from a marsupial-specific retrotransposition event. DNA Res. 18, 211-219. doi: 10.1093/dnares/dsr012
Ono, R., Nakamura, K., Inoue, K., Naruse, M., Usami, T., Wakisaka-Saito, N., et al. (2006). Deletion of Peg10, an imprinted gene acquired from a retrotransposon, causes early embryonic lethality. Nat. Genet. 38, 101-106. doi: 10.1038/ng1699

Ono, R., Shiura, H., Aburatani, H., Kohda, T., Kaneko-Ishino, T., and Ishino, F. (2003). Identification of a large novel imprinted gene cluster on mouse proximal chromosome 6. Genome Res. 13, 1696-1705. doi: 10.1101/gr. 906803

Roth, S. Y., Denu, J. M., and Allis, C. D. (2001). Histone acetyltransferases. Annu. Rev. Biochem. 70, 81-120. doi: 10.1146/annurev.biochem.70.1.81

Rupp, R. A., Snider, L., Weintraub, H. (1994). Xenopus embryos regulate the nuclear localization of XMyoD. Genes Dev 8, 1311-1323. doi: 10.1101/gad.8.11.1311

Sasaki, T., Nishihara, H., Hirakawa, M., Fujimura, K., Tanaka, M., Kokubo, N., et al. (2008). Possible involvement of SINEs in mammalian-specific brain formation. Proc. Natl. Acad. Sci. U.S.A. 105, 4220-4225. doi: 10.1073/pnas.0709398105

Schmidl, C., Klug, M., Boeld, T. J., Andreesen, R., Hoffmann, P., Edinger, M., et al. (2009). Lineage-specific DNA methylation in T cells correlates with histone methylation and enhancer activity. Genome Res. 19, 1165-1174. doi: 10.1101/gr.091470.109

Sekita, Y., Wagatsuma, H., Nakamura, K., Ono, R., Kagami, M., Wakisaka, N., et al. (2008). Role of retrotransposon-derived imprinted gene, Rtl1, in the feto-maternal interface of mouse placenta. Nat. Genet. 40, 243-248. doi: $10.1038 / \mathrm{ng} .2007 .51$

Smit, A. F. (1993). Identification of a new, abundant superfamily of mammalian LTR-transposons. Nucleic Acids Res. 21, 1863-1872. doi: 10.1093/nar/21.8.1863

Takemoto, T., Uchikawa, M., Yoshida, M., Bell, D. M., Lovell-Badge, R., Papaioannou, V. E., et al. (2011). Tbx6-dependent Sox2 regulation determines neural or mesodermal fate in axial stem cells. Nature 470, 394-398. doi: 10.1038/nature09729

Terasaki, H., Murakami, R., Yasuhiko, Y., Shin, I. T., Kohara, Y., Saga, Y., et al. (2006). Transgenic analysis of the medaka mesp-b enhancer in somitogenesis. Dev. Growth Differ. 48, 153-168. doi: 10.1111/j.1440-169X.2006.00853.x

Vasicek, T. J., Zeng, L., Guan, X. J., Zhang, T., Costantini, F., and Tilghman, S. M. (1997). Two dominant mutations in the mouse fused gene are the result of transposon insertions. Genetics 147, 777-786.

Waterston, R. H., Lindblad-Toh, K., Birney, E., Rogers, J., Abril, J. F., Agarwal, P., et al. (2002). Initial sequencing and comparative analysis of the mouse genome. Nature 420, 520-562. doi: 10.1038/nature01262

White, P. H., and Chapman, D. L. (2005). Dll1 is a downstream target of Tbx6 in the paraxial mesoderm. Genesis 42, 193-202. doi: 10.1002/gene.20140

Yagi, T., Tokunaga, T., Furuta, Y., Nada, S., Yoshida, M., Tsukada, T., et al. (1993). A novel ES cell line, TT2, with high germline-differentiating potency. Anal. Biochem. 214, 70-76. doi: 10.1006/abio.1993.1458

Yasuhiko, Y., Haraguchi, S., Kitajima, S., Takahashi, Y., Kanno, J., and Saga, Y. (2006). Tbx6-mediated Notch signaling controls somitespecific Mesp2 expression. Proc. Natl. Acad. Sci. U.S.A. 103, 3651-3656. doi: 10.1073/pnas.0508238103

Yasuhiko, Y., Kitajima, S., Takahashi, Y., Oginuma, M., Kagiwada, H., Kanno, J., et al. (2008). Functional importance of evolutionally conserved Tbx6 binding sites in the presomitic mesoderm-specific enhancer of Mesp2. Development 135, 3511-3519. doi: 10.1242/dev.027144

Yoder, J. A., Walsh, C. P., and Bestor, T. H. (1997). Cytosine methylation and the ecology of intragenomic parasites. Trends Genet. 13, 335-340. doi: 10.1016/S0168-9525(97)01181-5

Conflict of Interest Statement: The authors declare that the research was conducted in the absence of any commercial or financial relationships that could be construed as a potential conflict of interest.

Copyright (C) 2017 Yasuhiko, Hirabayashi and Ono. This is an open-access article distributed under the terms of the Creative Commons Attribution License (CC BY). The use, distribution or reproduction in other forums is permitted, provided the original author(s) or licensor are credited and that the original publication in this journal is cited, in accordance with accepted academic practice. No use, distribution or reproduction is permitted which does not comply with these terms. 